\title{
Contextualized mobile game-based learning application for computing education
}

\author{
Alok Kumar Yadav ${ }^{1}$ (D) Solomon Sunday Oyelere ${ }^{1}$
}

Received: 24 July 2020 / Accepted: 23 October 2020/ Published online: 4 November 2020

(C) The Author(s) 2020

\begin{abstract}
This paper intends to present an overview of a mobile game-based learning application, BaghLearn that develops and upskills programming and algorithmic knowledge by cross-curricular capabilities through a traditional world-based game. The focus of this research was to explore the learning effectiveness of BaghLearn on students. Mixed method research approach was applied to collect, process and analyze the research data in which undergraduate students who had some prior knowledge or had taken algorithm courses were instructed to test the mobile game learning application. This study exhibits the idea of integrating learning with contextual mobile game as an effective approach in understanding the influence of games towards cognitive achievements of students in computing education. In addition, there are no major requirements for the use of this application (can be maintained in resource-constrained contexts such as Nepal), which makes it expressively satisfying and useful for students who are relentlessly using mobile devices. Besides, this study evaluated the influence of BaghLearn towards learning of the design and analysis of algorithm course, which is a compulsory course for most undergraduate computing education program. Furthermore, the study findings can be used as a guideline for developing learning solutions and usability evaluation of such solutions, especially for infrastructure-constrained contexts. Students using the BaghLearn opined that the application is easy to use, supportive and lead to improved learning satisfaction.
\end{abstract}

Keywords BaghLearn · Computing education · Design and analysis of algorithms · Gamebased learning $\cdot$ Nepalese higher education system $\cdot$ Usability evaluation

Alok Kumar Yadav

aloky@uef.fi

Solomon Sunday Oyelere

solomon.oyelere@uef.fi

1 School of Computing, University of Eastern Finland, FIN-80101 Joensuu, Finland 


\section{Introduction}

Learning can be viewed as an activity that fosters knowledge and skills. For the past few decades, there has been a shift in the learning paradigm. It is now associated with a broader combination of behavioral and cognitive construct while making it interesting and engaging. Therefore, (Prensky 2002) suggested a better approach of learning through a real game play so that students along with having fun can learn with better effectiveness. Digital technology on the other hand has been a driving factor in supporting digital learning platforms that will enable learners with efficient and personalized learning experiences along with providing a freedom for learning and determination for collaboration that are beyond traditional learning (Sari and Tedjasaputra 2019). Digital devices such as tablets, smartphones and many others are increasing making influence among educators and students (Kachur et al. 2013; Anohah et al. 2017) due to the fact that these mobile devices have gained popularity, especially in low-tech or infrastructure-constrained developing countries such as Nepal, and possess enhanced capability, and more enhanced low cost features. Also, the outcome of the students learning performance are strongly positively linked to the effective use of technology in the educational environment (Alkhalifah et al. 2017). Furthermore, the introduction of technology is seen to have a collaborative advantage, promoting greater communication between teachers and students and enabling students to express their views more often (Vasudeva et al. 2017). With all these considerations, mobile devices for no doubt have been used for learning purposes. At the same time, mobile devices can be best employed with collective learning because of the fact that learners can have full control over the device without any obstruction from complex tools, allowing them to have complete liberty in their own learning environment and unrushed with their own pace and timing (Parsons 2010; Shonola et al. 2016). In addition, (Schmitz et al. 2015) stated that many educational professionals and scientists have commonly acknowledged the usage of mobile devices to involve learner and support learning. Out of the different formats used commonly to advantage mobile technology, usages of mobile games for learning is one of them. Nowadays, game based learning is used as technique and resource in the classroom to enhance learners' interest, since, most of the educationally designed games often generate player enjoyment which in return benefit educational objectives (Daungcharone 2016). Specifically, game based learning will establish a harmony between gaming and learning elements, thereby building two essential foundations: fun component and educational component (All et al. 2016). As such most lecturers use game based learning strategy to enhance both learning and engagement in their studies (Tsai et al. 2017). In game based learning, learners compete in a gaming scenario while attempting to execute a set of learning activities on their own or in a group. (Tlili et al. 2016b) realized that game-based learning integrates the element of immersion that does not occur in classroom. Not just that, but such games often endorse adaptive learning as well by developing model (Khenissi et al. 2015; Tlili et al. 2016a).

This study aims at bridging the gap between learning efficacy and motivational appeal with the support of a mobile game-based application, BaghLearn along with exploring the effectiveness of game-based learning towards improving the perception of students towards learning, participation and collaboration (Hartt and Mostafapour 2019). Originally, BaghLearn was developed from a well-known traditional game in the context of Nepal, Bagh-Chal. BaghLearn will not only support gaming, but also learning algorithmic concepts in the Nepalese higher education context since games are 
believed to support the prospect of learning of academic subjects easier, more entertaining, more learner-centered and even more efficient if encompassed with subject matters (Resnick et al. 2009; Prensky 2002). There is a proof that well designed educational games not just maintain the motivation of the students to learn better than their conventional counterparts, but also improves the learning result (Zhu et al. 2019). The notion of mobile game-based learning in the education mainly centers on the meticulous work of game development process, a physical solution is proposed to solve the problem that relates to real-life context which may include theoretical learning content (Emerson et al. 2018). Bagh-Chal is a strategic, two-player board game that originated in Nepal, and very popular in the rural areas, which has little to none technology infrastructure. In our illustration, the content is algorithmic concepts in Nepalese higher education. In essence, this study extends the entire idea governed from development of artifact, evaluation to improvement of game-based learning in a repeated manner using the resources available locally (technology and proficiency) (Haruna et al. 2019) and with higher level of digital literacy of the learning population. Corresponding to which the features of the game-based learning is maintained low profile in contrast to those that are deployed in high tech settings. Thus, a less sophisticated game-based learning platform was developed considering wider perspective of the population using a blender learning approach. Since the traditional learning method is abominating in front of modern methods of education, there is consistent need of reform in education technology even in Nepalese education system that would make learning more effective, highly motivating, gain knowledge, more interesting and engaging (Haruna et al. 2019).

The main objective of this research is to understand the opinion of the students towards the usability and effectiveness of mobile game-based learning application. The usability evaluation is conducted using the USE (Usefulness, Satisfaction, and Ease of Use) questionnaire (Lund 2001). The USE instrument has been used widely for evaluating new information system and comprises these four elements: usefulness, ease of use, ease of learning and satisfaction that are suitable to understand the perception of Nepalese higher education students, and examine the exposure they have in their studies with regard to game-based learning platform. This study will answer the following research question:

Research Question 1. To what extent do the students find the BaghLearn mobile game-based learning application to support computing education usable?

Apart from the objective, the overall goal of this study is to improve computing education, more specifically algorithmic education, by ensuring that students have better learning opportunities and options than by solely dependence on traditional learning method (Haruna et al. 2019; Carvalho et al. 2015).

\section{Background}

\subsection{Nepalese context for Mobile learning}

Precisely, mobile learning is relevant to handheld electronic devices that can be accessed anytime and anywhere (Kukulska-Hulme and Shield 2008). Although mobile devices are the main instrument of mobile learning, there are other activities that itself 
define the course of action that involves learners, teachers, environment of learning, learning theories and all other activities that relates to it (Sharples et al. 2007; Oyelere et al. 2018). Mehdipour and Zerehkafi (2013) mentioned in their article that mobile learning has the capability to enhance literacy level, numbers and participation of young students in education. Likewise, they are useful for learning in both the context, formal and informal since they share content knowledge which is useful in retaining it for longer duration (Hartt and Mostafapour 2019). Research projects have predicted better end results in both formal and informal sector with mobile learning (Hayati et al. 2013; Tewari et al. 2010). In Nepalese higher education context, mobile learning can support significant changes and that too with a game-based learning where students will learn to become competitive, not with other students but against the game where to score higher they have to learn better. Similarly, it consists of game-based dynamics, design and strategic theory that involves users, encouraging action, promoting learning and solving problems which ultimately helps to recall certain facet and retaining few analytical chronologies that improves in better retaining of knowledge (Kadel et al. 2018).

The acceptance of mobile based learning has been at rise in informal learning, but this has gained very little consideration. The mobile penetration rate was $98 \%$ by the end of November 2019, data released by Nepal Telecommunication Authority in January 2020 (NTA 2020) which shows that young people with majority of university students make-up of the bigger portion of mobile subscription thus providing better and immense opportunity of mobile learning among Nepalese students. Furthermore, Tribhuvan University (one of the largest governing university of Nepal) has pushed itself to incorporate information and communication technology (ICT 2013) to study syllabus of higher education, has started Centre for Information Technology (CIT) and Information and Communication Technology Center (ICTC) in order to integrate hybridizing traditional education programs as a measured development to virtual learning (ODEC-TU 2015). Other than that, the ICTC Center has also developed an android application to support learning (Parajuli 2016), therefore initializing the use of mobile learning in Nepalese higher education context. With all these developments, there has been an emerging acceptance among students while bridging the digital divide by offering an alternative technology for learning (Parajuli 2016).

\subsection{Related game-based Mobile learning solutions in computing education}

Game based learning is about educational games that would improve student's attitude towards learning and approach of learning with respect to students. The goal is for students to appreciate the learning process itself. Hence games are implemented to improve the learning experience thereby proving a balance between the learning unit and the games and their real-world implementation (Kadel et al. 2018). Mobile based games are mostly remodeling of popular computer-based games that are kept simple with entertainment-oriented purpose. Considering that games are linked to teenagers' growth process (Kinshuk and Chen 2005) and also pondering the fact that game-based learning could be an essential alternative to old school method (Antonellis et al. 2005), mobile game-based learning in present is contextualized. Several studies have been executed with the developed game which intends to incorporate relevant features that favors learning on the computational domain of which some aiming to cover particular 
units while others sighting for diverse learning goals and units (Oyelere et al. 2017). Colobot (2007) is well known as the first computing game that is commercially available to teach programming where players use the list of commands available as a pseudo code (similar to $\mathrm{C}++$ ) to move game objects in order to complete the tasks. Though being a fun learning game, it may be customized according to the spectrum of the curriculum. Similarly, development of CMX (Malliarakis et al. 2013; Malliarakis et al. 2014) which emphasized on improving the learning aspect while using it in the classroom as an assistance tool by upscaling student's engagement in order to enact more on the concept they learned within the supervision of a tutor. Several others, Saving Serra (Richter et al. 2007), EleMental (Doran et al. 2009), MobileEdu puzzle (Oyelere et al. 2017) and Catacombs (Richter et al. 2007) that are specially designed to teach programming with different gaming elements. CodeMonkey (2018), another web-based game that enhances computing focusing mainly on problem statements rather than thinking on complex computing syntax. It even has tutoring on topics if the learner faces any difficulty or hurdle. Likewise, Lightbot (2017), a multi-platform game, that uses robot as a game object to solve problems and advance it to the next round. This game uses the coding concept like statements loops and procedures but without using programming syntax. Parallel (Zhu et al. 2019), an educational game that was designed to teach key Computer programming concepts. This game introduces a completely new design space framework focused around how the player writes the algorithms and how they are implemented in the game. It is clear that there are many game based learning solution that are built to promote computing education or to promote computer science courses but it is also evident that these solutions lack involvement and fun that would potentially offer course information along with ability to engage with material (Lepp et al. 2015).

From the studies it is evident that game-based learning conceptualizations and contextualization are encouraging and motivating students through a game can be convincing and even have implications in developing computing skills. Hence, a comprehensible solution should come up connecting a game that actually support learning along with strengthening ideas on the subject matter thus contextualizing the importance of interrelation between the game play and knowledge. With all this in consideration, BaghLearn has tried to strengthen the interrelation between actual game play and learning thus creating a much-needed involvement of students in learning key algorithmic concepts.

\subsection{Learning theories in computing education}

Research in computer education is focused on theoretical constructs, particularly unique to computer education and more specifically based in learning, frame of mind, and certain other factors. Besides being a subjective action, learning is dependent on the processing power, commitment and extent of the learning information processes by the learners. Interpretation of learning content allows students to understand better. Furthermore, students learn better when allowed to extract tangible values from learning material and collaborate with other learners. The knowledge of analyzing and specifically integrating theoretical concepts into study is constantly growing in the field Computer Science Education (Daniels and Pears 2012). In educational research, theories and concepts on how people study plays a significant role since they not only 
influence the research questions and helps selecting the type of data collection and analysis technique but even significantly influence the development of pedagogical concepts and approaches (Knobelsdorf 2015). The current learning framework appears to see learning as the development of abilities and skills by a person where the instructor serves as nothing more than a facilitator. This research will primarily concentrate on learning theories that are closely related to and frequently linked to computer science education including mobile learning like Constructivism, Connectivism, Cognitivism and Behaviorism. These learning theories are chosen since they embrace the essence of most of the topics of computing education and provide the fundamental ideas in the modern age to assist learning with the help of mobile devices.

While behaviorism concentrates on actions that encourage learning as a tangible shift in the person's noticeable actions or that are promoted by enhancing a particular stimulus and reaction (Zendler 2018), It plays a critical part in fulfilling the standards of learning. The learners therefore appear to replicate a behavior that generates positive feedback (Oyelere et al. 2017). Relating to this research, the action of behaviorism would be test questions and evaluations of those questions.

Cognitivism on the other hand is associated with a person's capacity to think critically about knowledge in the learning process (Taylor et al. 2013). With respect to behaviorism cognitivism puts emphasis on the exploration of the learning process and more specifically on occurrence of understanding, problem solving and reasoning (Keskin and Metcalf 2011). With respect to this research, cognitivism should be on knowledge and content delivery to facilitate learning outcomes through the use of image and learning windows.

Connectivism is primarily involved with cognitive growth focused on perceiving in addition to physical growth and development through the exposure of an individual to the social environment. It is especially important in which it involves the interpretation of behavioral success and ethical formation in diverse contexts (Rice 2018; Goldie 2016). Therefore, accessing information and resources anywhere and at any time through mobile devices correlates to the Connectivism theory of this research.

Lastly, constructivism learning theory, which is also the theoretical framework for this research work deals with integrating new knowledge together with what they already know (Bada and Olusegun 2015). In the course of knowledge acquisition, this theory considers learners as an active participant where the learner deliberately creates insights rather than consuming it ineffectively from classrooms and lectures which is also the case in our study i.e. with BaghLearn students themselves are involved in the thought process and themselves develops their understanding and knowledge. In the process of assisting learners, BaghLearn helps learners develop new insight from their experience. When put in acceptable environments, constructivism anticipates learners to perceive knowledge independently and so by themselves. The same is with BaghLearn where the learner is brought to an environment that is independent and the player advances through the game with his perceived understanding from the learning module. Furthermore, BaghLearn occurs in a practical environment since constructivism theory has an environmental influence and immersive learning experience as an essence for good learning. Constructivism considers learning based on social connections, teamwork and practical evaluation of students which holds the notion for social constructivism that people do not gain knowledge only just to read, write and communicate but rather to express in different ways for various reasons particularly in 
social settings (Guo 2018). The second notion of constructivism that immensely relates to this study is that learning here is active i.e. learners challenge their knowledge base in view of what they come across. If the current understanding is unfamiliar then learners can adjust their understanding to reflect new experiences based on the assessment and yet the learner stays active throughout the action.

\section{BaghLearn game implementation}

BaghLearn is a game design made with blending of traditional world-based gameBaghchal with some serious learning on various topics of Design and Analysis of Algorithms within the domain that distinctly favors the attainment of computational education. It is an Android-based mobile application developed with the use of Unity Development Engine version 2017. The application is built in Android platform because it is open source, which means no license, distribution and development fee and even supports a wide range of platforms, Windows, Mac, and Linux. Each module of the application has been built separately to ensure the dynamic nature along with high end productivity (with little effort students can learn better), portability (can learn from anywhere and anytime) and maintainability (easy to correct errors and progress accordingly). Each module is then combined using scene management library for high end performance of the application. Each module has been tested thoroughly for numerous times before they are finally consolidated. Even after the final merger, application is tested numerous times in the emulator and on actual devices to verify the performance and integration of different modules. The application is tested on a OnePlus 6 mobile device with OS version 10.3.0 and display size of 6.28 in. (runs comfortably on older version as well) for debugging if there are any in real time scenario. Application is tested iteratively and with fine precision.

BaghLearn mobile game-based design application is a multiplayer touch-based 3D game with a novel approach blended between learning and playing. This application not only creates a mentality that inspires students to try new things but not to be worried of failure and also to empower them to participate in fun learning experiences (Lee and Hammer 2011). In addition, BaghLearn has adequately tried to answer the way young people learn today and engage them in a more efficient and effective manner. This game design supports the four traits of modern learning that are portability, social interaction, connectivity and individuality which can be adapted to needs of individual learners (Huang et al. 2017). Apart from this, BaghLearn is totally immersive in nature in that this Learning application engages learners with little to no consciousness about the environment around them. The main motive of blending a game to the learning is that the game triggers factors like interest, fantasy, competitiveness, coordination, recognition, influence and challenge (Hamari et al. 2016).This learning application can be used without the use of internet connection (a suitable characteristic for a resource-constrained content such as Nepal). The Home Screen (See Fig. 1) features four buttons aligned centrally with one at the top right corner. Both instructor and learner can opt for any of the buttons before the actual game play. Students can Learn about the game rules from About button, set the parameters from option button and select topic of learning from top right button that will redirect to the learning module. Fig. 2 presents the topic screen showing various topics currently available in the application. Once having enough knowledge on that topic, students can go with the play button to start the game. 


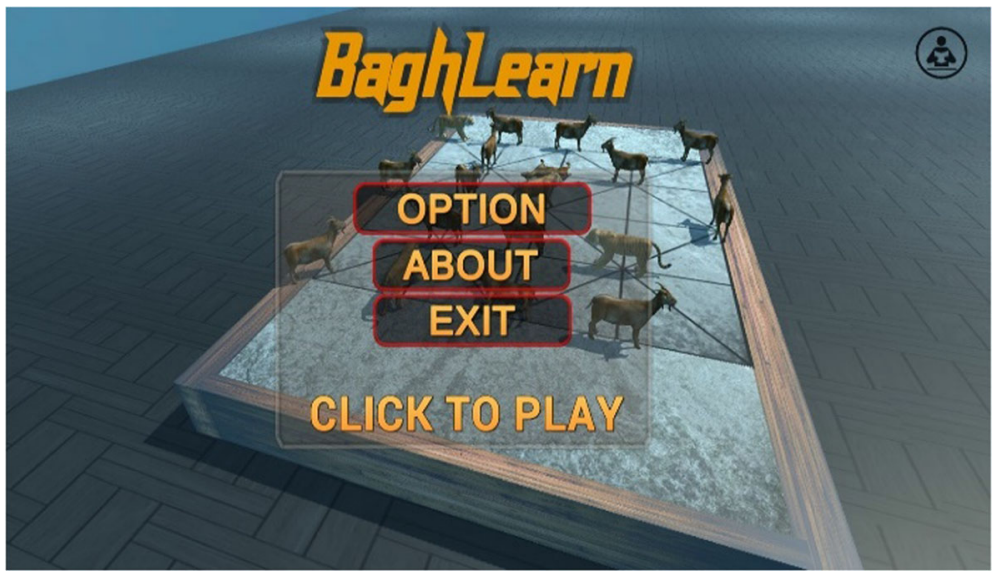

Fig. 1 Home screen of BaghLearn mobile game-based application

The BaghLearn mobile game-based application interface contains players turn, number of tiger player captured, number of Goat player killed in order to showcase the winning player.

In normal game scenario, there are twenty goats and four tigers game players but with this game those numbers vary with learning outcome. There are lists of subjects to choose from and with each chosen subject students learn basic ideas and working principles on the topic (see Fig. 3) followed by knowledge testing. These learning includes all the information students need to get familiar with the topics. It is a plus if a learner has a background knowledge of computer algorithms but not required, ideas covered on application are enough to advance.

\subsection{Integration of learning module into gameplay}

The fundamental idea is to educate students with the basic concepts of design and analysis of algorithms with Baghchal game. BaghLearn application presents a learning construct where learners are first presented with a basic overview of the topic and then

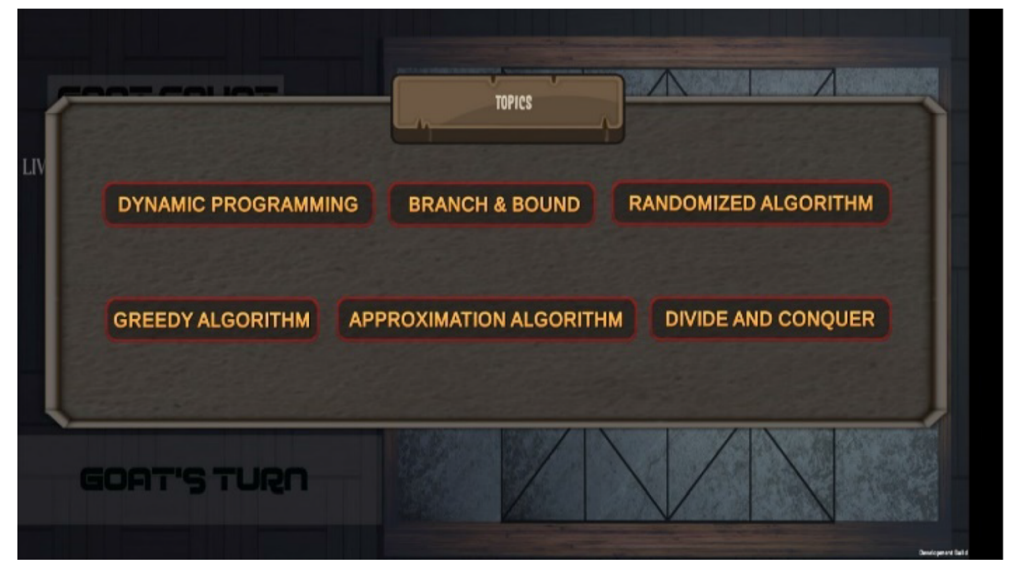

Fig. 2 The topic screen showing different topics of design and analysis of algorithms in computing education 


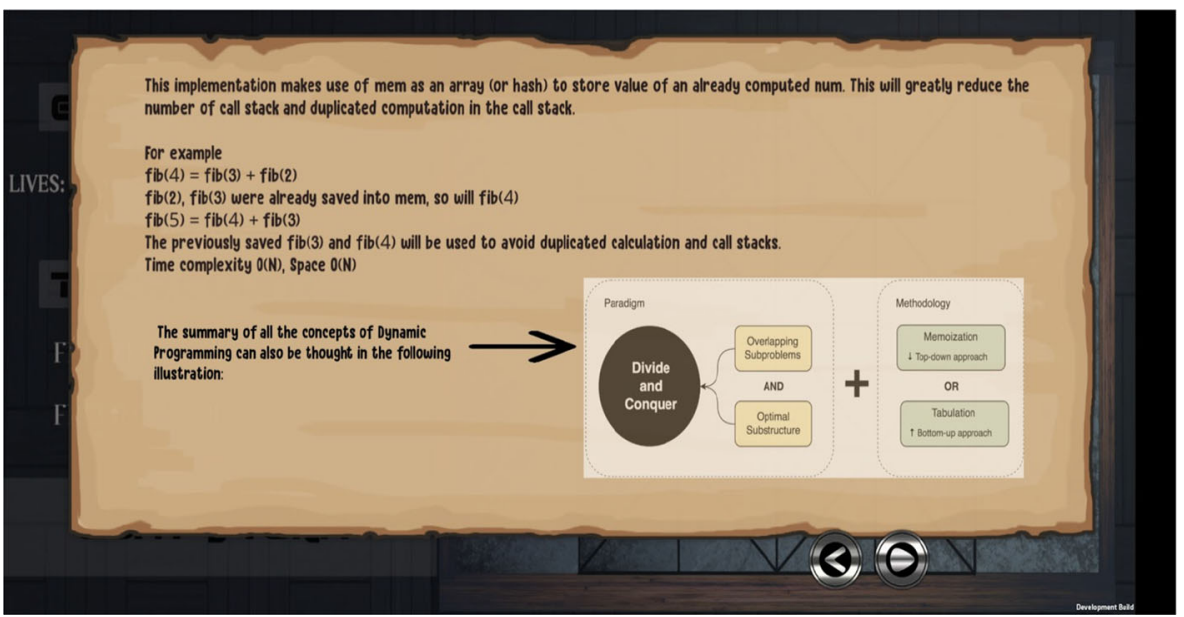

Fig. 3 First slide on selected topic

with a set of fragments of code, block or a single lines of text, multiple type question that are related to algorithms. As part of a learning, students thus choose the right option based on the calculation made thereafter gaining understanding and concepts of algorithmic logic. The purpose of the action is to make students read and understand the problem statement, deduce reasoning and decision making ability to solve the problem. The learners' quiz interface is presented in Fig. 4. The quiz interface also consists of a timer (top right) and score count (top right). If in case problem is not solved in the given time frame, the player loses the chance to answer the question and is awarded no points. The next round of question appears immediately after the player makes a choice or if the time elapses. For the feedback, the interface responds instantly after each correct answer. If the answer is correct, 10 points add on to the score list and if the answer is incorrect, nothing happens to the score. At the end of the quiz play, total points scored in the round is shown for each topic chosen as shown in Fig. 5. Each quiz topic contains multiple sets of questions and each round (tiger and goat) has 6 questions on each with

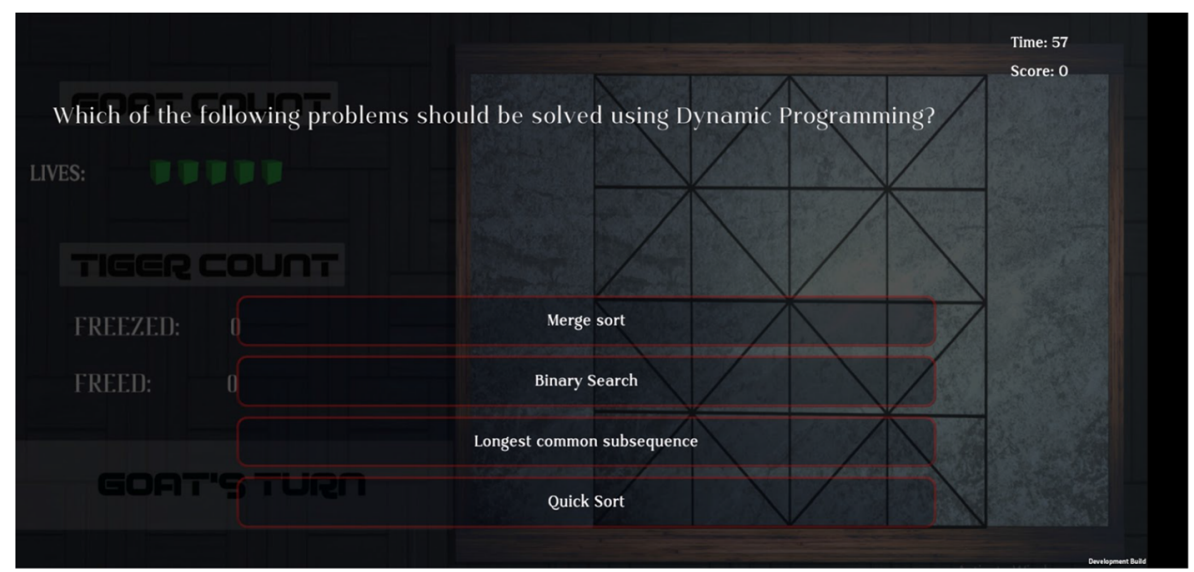

Fig. 4 An example of the quiz interface showing multiple-choice options. A countdown timer and player score can be seen on the top right corner of the application 


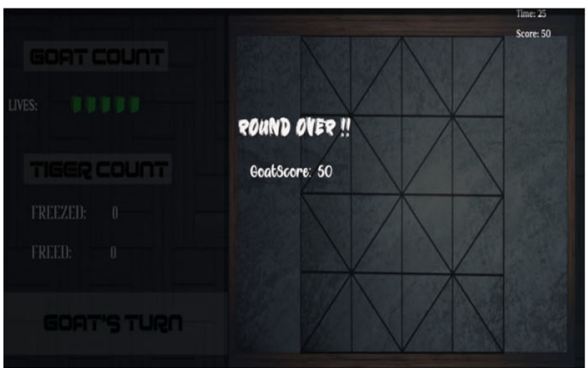

a

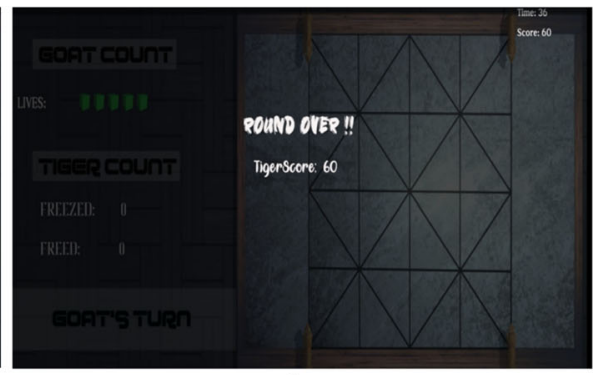

b

Fig. 5 Total points scored by two players

time limit and score that adds up with each correct answer. Learners should score high to make game play more interesting and involving. With each correct answer the player on board has a higher chance to triumph.

Figure 6 illustrates an image of the 3D game interface of BaghLearn. Number of game players depends on points scored by students in the quiz section. For example, in the case of a Tiger player, if the score is 40, 50 and 60, then goat game object generation is 19, 18, and 17 respectively, a dynamic approach to involve learner. Similarly, if point scored is 50 and 60 for goat, then Tiger game object generation is 3 and 2 respectively.

All players can be hauled from their position and placed into specific slot defined by the game rule. The game is started by a Goat player first, since it is a multiplayer game, Goat move is followed by Tiger move. Players can choose between their pieces for movement in any succession, for as long as they are allowed to move. From the above game settings, this research intends to come up with a model, where working of knowledge maturity of one player which can be notably distinct to that of the another player or even may be related to the same maturity stage who is at the identical level unites their learning. The conceptual working method of BaghLearn system using a use case diagram is depicted in Fig. 7.

\subsection{Few examples of learning construct}

Example 1: Here, the task of the player is to calculate the number of ways in which the given denomination of coins can be arranged to sum the value 7 . The example will

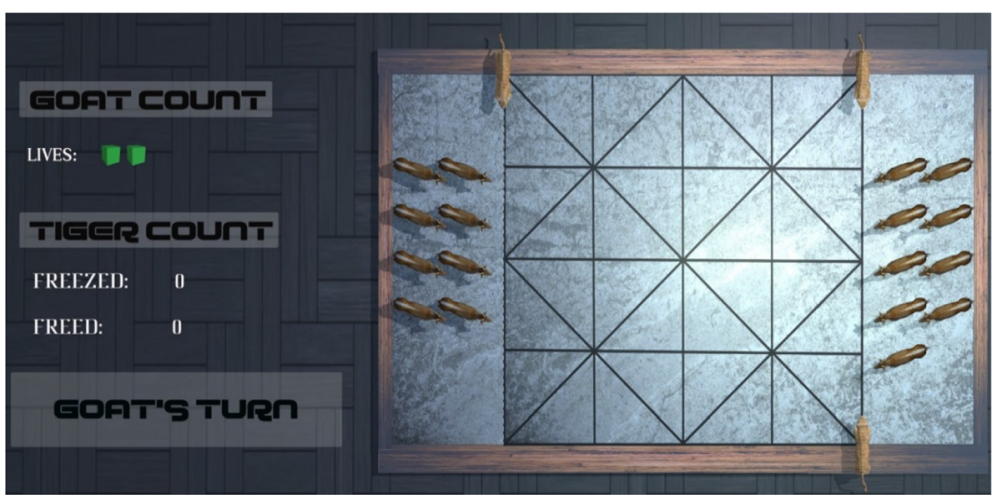

Fig. 6 BaghLearn game play interface 


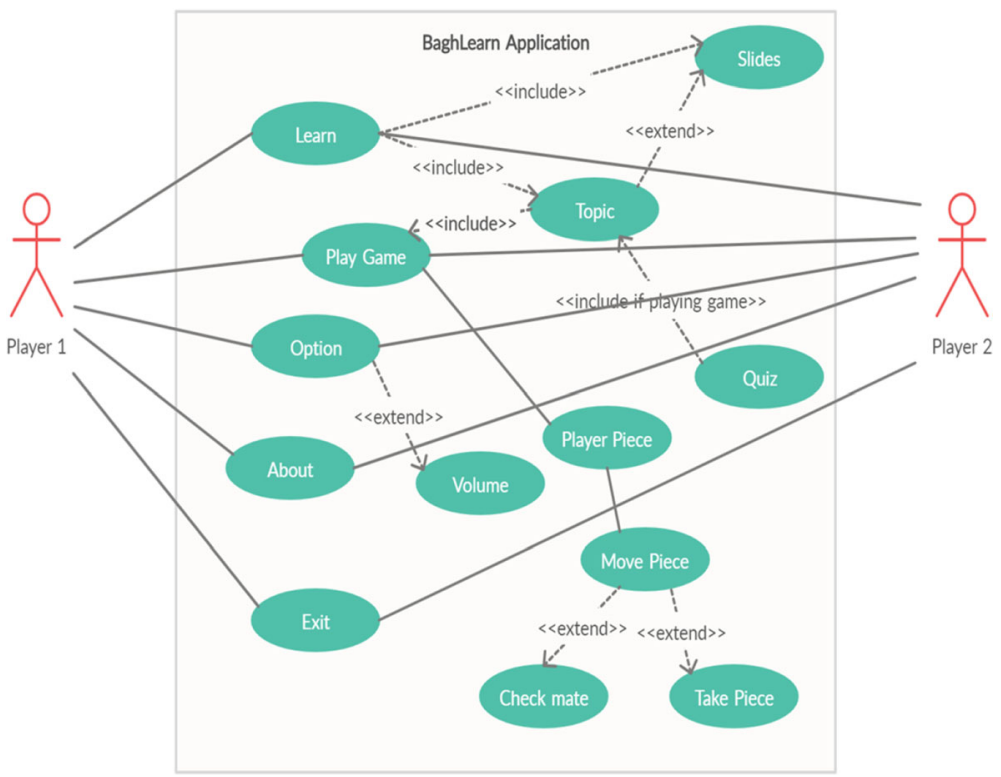

Fig. 7 Use case diagram for BaghLearn mobile game-based application

guide learner to construct a method using Divide and Conquer along with his ability to sustain concentration on a subject matter with better manipulation ability. Fig. 8 shows the task interface for this example.

Example 2: Here, player's task is to identify the best possible algorithm that matches the given statement. With this question learner will be able to precisely differentiate between different algorithms and the ability to break complicated tasks into smaller tasks and assign them in the right order. Fig. 9 shows the task interface for example 2.

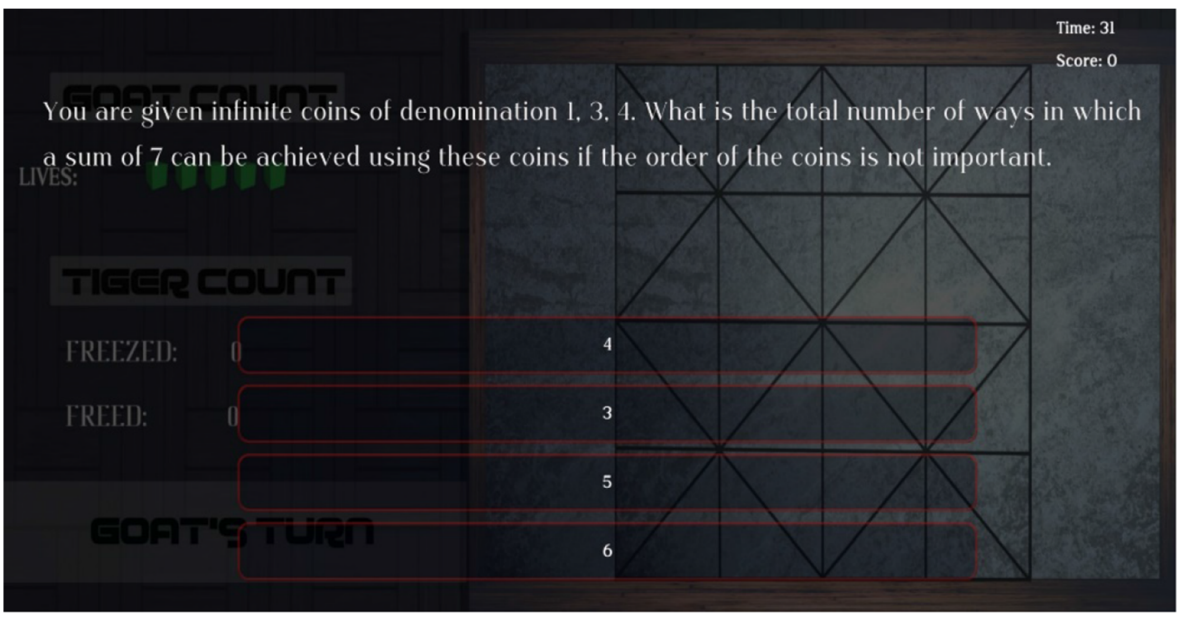

Fig. 8 Indicating the description of the task for example 1 


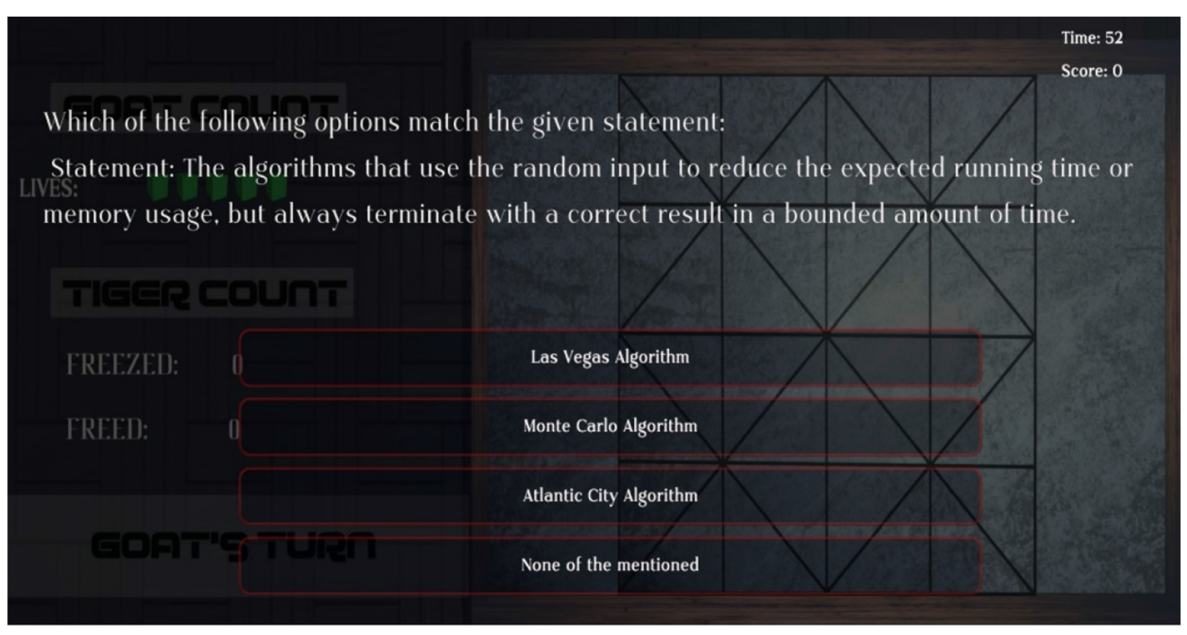

Fig. 9 Indicating the description of the task from example 2

\section{Methodology}

\subsection{Research design}

The developed learning tool was tested to evaluate feasibility, effectiveness and suitability (Oyelere et al. 2018; Shyshkanova et al. 2017) as a part of a practical study that was conducted during the educational process in a Nepalese higher education system. The aim of this practical research was to apprehend on how this BaghLearn game is perceived by the students of undergraduate studies and if it has procured the usability perception of the learners and support them obtain better results than the traditional old school method (Oyelere et al. 2018). This research employed a sequential explanatory mixed method design, comprised of two different phases (Creswell and Clark 2017). First quantitative numeric data is collected and analyzed in this method, followed by qualitative data collection which helps to explain or expand on the quantitative findings obtained in the first phase. The purpose for collecting both quantitative and qualitative data is to ascertain whether the response of perceptiveness of the distributed undergraduate students from quantitative phase vary from individual viewpoints and, if so, to consider why such responses and viewpoints may vary. Therefore, the quantitative data and finding offered a general description of the research question, whereas the qualitative data and its interpretation clarified that statistical finding by examining the perspective of the participants in detail. A survey based on the USE questionnaire was conducted as part of quantitative data collection that served close ended questions followed by semi-structured interview where student selection was done by judgmental sampling (Parajuli 2016) which further helped to analyze and explain the inclination and complications that arise concerning the open ended section of the questionnaire. The interview will further help to estimate the perception and viewpoint towards game-based learning, BaghLearn application. The qualitative approach was given priority in the study because, it concentrated on the in depth interpretation of the findings obtained from the first i.e. quantitative phase and also because of the two level case review. Later, both qualitative and quantitative phases were aligned to develop a concrete results of the whole study. 


\subsection{Target participants}

The study was conducted on one of the affiliated campuses of Tribhuvan University, Institute of Engineering, Kathmandu, Nepal. The sample group includes 42 on-campus third year undergraduate students conducted on the design and analysis of algorithms course, which is a compulsory undergraduate course in computing education. The participants were subjected to both methods of learning (traditional and game-based) based on defined course structure. Participants were first put to learn with a traditional method followed by the BaghLearn Mobile application. After the traditional learning method, participants were presented with a brief lesson on game programming and that too with ground information on game-based learning and its effectiveness. The entire research process is illustrated in Fig. 10.

\subsection{Data collection and analysis method}

In the first stage of execution, students from the course Design and Analysis of Algorithm were introduced to the topics that were relevant as a part of our learning application on the basis of design analysis (Oyelere et al. 2018) and also significant to the university course structure. Additionally, students were given a brief introduction on game-based learning, its core concept of repetition based learning, its failures and accomplishment of goals and how that takes the applied concept to teaching a curriculum. Students were also provided with the user guide of the BaghLearn game-based mobile learning application.

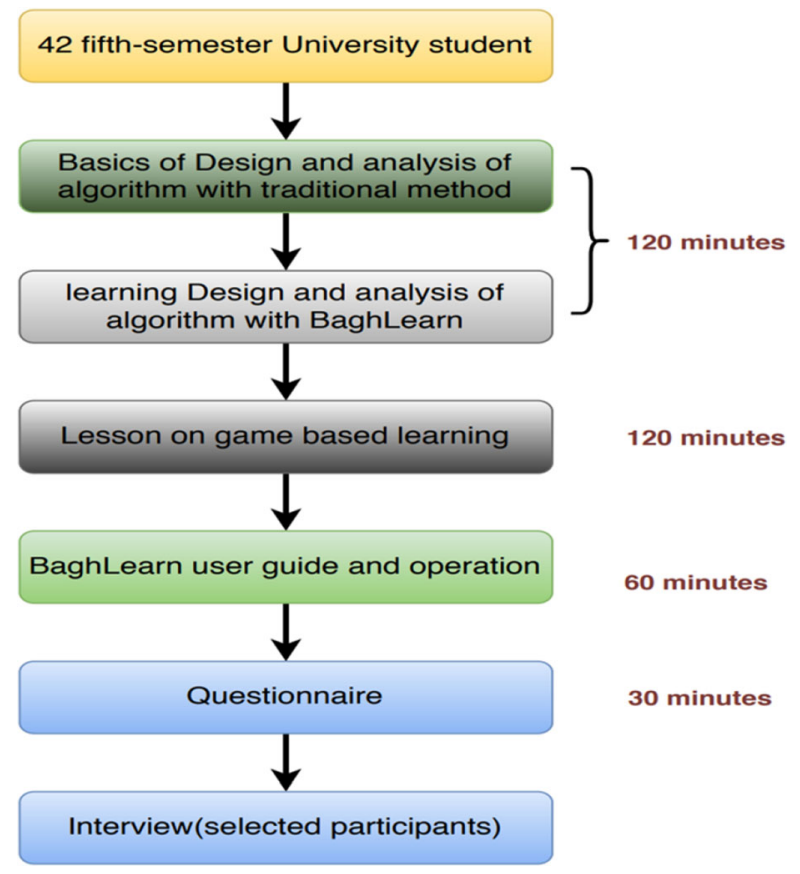

Fig. 10 Research process of evaluating the BaghLearn application 
In the next stage of execution, after having a traditional learning method, students were subjected to game-based learning on the BaghLearn application. The contents of learning for both the methods were the same. For the game-based learning, learning along with the quiz portion and game play of the application BaghLearn was used. For traditional learning method, face to face learning with brief scientific texts on the subject matters that contained suitable picture and descriptions were used where students were directed to carry out their studies in the same manner as they were used to in their regular classes (Rondon et al. 2013). In the game-based learning, topic support along with self-study quizzes based on numerous topics were aided in a single platform to learn and enhance understanding. A mixed method research process was undertaken in this study. For the first quantitative research process, to evaluate students' performance and the effectiveness of the application, the participants were put forward with some questions in an assessment sheet. The questionnaire accommodates 29 items which were systematized and topically structured in 4 areas (Bartel and Hagel 2014): the usefulness of BaghLearn (8 items), Ease of Use (11 items), Ease of Learning (4 items) and Satisfaction (6 items). A seven-point survey scale representing a set of answer marks was used, so to confirm the statement they decided with "strongly agree ", "moderately agree", "agree", "undecided", "slightly disagree"," disagree"," strongly disagree". For the qualitative research process (Creswell et al. 2006), in-depth semi structured interviews with 10 participants was organized. Since the purpose of the quantitative phase is to analyze and expand on the finding of the statistical experiments, there was a need to understand why a certain variable responded differently to the role with respect to their consistency and for this reason four open-ended questions were asked in order to examine the position of these four variables (learning environment, self-motivation, learning experience, student support), demonstrating significantly important predictive capability for the samples. The analysis of questionnaire data was processed using Microsoft excel sheet and SPSS tools, while the qualitative data was analyzed using content analysis through transcription, coding, and thematic representations (Creswell and Clark 2017). For the detailed data analysis simple descriptive statistical values like mean and standard deviation were calculated to understand the opinion of the students regarding the usefulness of the BaghLearn application to support computing education.

\section{Results}

\subsection{Quantitative results}

The results from the USE questionnaire forms the quantitative part of the study, in which the students' response were analyzed to understand the usefulness, ease of use and ease of learning with the BaghLearn mobile game-based learning application.

Table 1 shows the usefulness of the BaghLearn application in learning design and analysis of algorithms. With a mean value of 6.12 and standard deviation of 0.942 , which on a 7 -point Likert scale ( $7=$ strongly agree; $1=$ strongly disagree $)$ is in the range of agree and strongly agree. Therefore, most of the students felt that the use of application has helped them to be more effective which was one of the key attributes while determining the requirements towards the development of BaghLearn. Besides, 
Table 1 Results indicating the usefulness of BaghLearn mobile game-based learning application

\begin{tabular}{lll}
\hline Statements & Mean & Standard Deviation \\
\hline Q1. It helped to be more effective & 6.12 & 0.942 \\
Q2. It helped to be more productive & 5.71 & 1.019 \\
Q3. It is useful & 6.67 & 0.570 \\
Q4. It give more control over the time activities in life & 5.07 & 1.177 \\
Q5. It makes the things one would want to accomplish easier to get done & 5.57 & 1.272 \\
Q6. It saves one's time when they use it & 5.48 & 0.917 \\
Q7. It meets one's need & 5.79 & 1.240 \\
Q8. It does everything I would expect it to do & 6.62 & 0.627 \\
\hline
\end{tabular}

with a mean value of 5.07 and a standard deviation of 1.177 , most of the participants agreed that the application provides balance over the activities in their life with few undecided that it would in any manner gives control over their activities. Thus, considering the depth in the attribute of the application, which in certain aspects impact the search time feature is certainly acceptable. Commendably the statement "It saves my time when I use it", "it is productive", "makes thing easier to get job done" and "it meets my need" has a strong mean value of 5.48 with a standard deviation of 0.917 , mean value of 5.71 with standard deviation of 1.019 , mean value of 5.57 with standard deviation of 1.272 and mean value of 5.79 with standard deviation of 1.240 respectively, thus falling response of the students' in the range of agree to moderately agree. Here can be seen a scattered data thus giving an appearance that there is a preference and interest among students towards their requirements.

Table 2 shows the data for easiness in operating the application. Most of the statements have a mean value over 6.0 thus falling in the range of moderately agree and strongly agree with more participants inclined towards strong agreement either be it the simplicity, easiness at operation, user friendliness, effortlessness or even the flexibility. Most of the section got a strong response of agreement from participants

Table 2 Results showing the ease of use of BaghLearn mobile game-based learning application

\begin{tabular}{lll}
\hline Statements & Mean & Standard Deviation \\
\hline Q9. It is easy to use & 6.60 & 0.627 \\
Q10. It is simple to use & 6.40 & 0.828 \\
Q11. It is user friendly & 6.57 & 0.590 \\
Q12. It requires the fewest steps possible to accomplish what I want to do with it & 6.52 & 0.671 \\
Q13. It is flexible & 6.10 & 1.078 \\
Q14. Using it is effortless & 6.55 & 0.670 \\
Q15. one use it without written instructions & 6.17 & 0.961 \\
Q16. one don't notice any inconsistencies as they use it & 6.62 & 0.731 \\
Q17. Both occasional and regular users would like it & 5.98 & 1.179 \\
Q18. One can recover from mistakes quickly and easily & 5.88 & 1.152 \\
Q19. One can use it successfully every time & 6.88 & 0.354 \\
\hline
\end{tabular}


with disagreement counting to 0 except for few (4 participants) where participants were unable to decide the adaptably feature. Most of participants (Mean 5.98 and standard deviation of 1.179) were in agreement that BaghLearn can be helpful for both occasional and regular users' while few stressed on using it on a more regular basis. Since the application is easy to use, most of the students with a strong mean value of 5.88 and standard deviation of 1.152, falling in the range of agree and moderately agree, find that it would help them improve and correct their mistakes with each iteration of game play. Likewise, the statement "It requires fewer steps to accomplish task", "can operate without instruction", "didn't notice any inconsistencies" and "using application successfully every time" has a strong mean value of 6.52 with a standard deviation of 0.671 , mean value of 6.17 with standard deviation of 0.961 , mean value of 6.62 with standard deviation of 0.731 and mean value of 6.88 with standard deviation of 0.354 respectively, thus falling response of the students' in the range of moderately agree to strongly agree. Such a strong response from participants further assures that application has a user-friendly, clean and simple UI keeping high performance for long term engagement.

The next section of analyzed result (Table 3) was about the handling of the application. For instance, the participants were asked if one learned to use it quickly in which the result obtained has a mean value of 6.19 and a standard deviation of 0.833 which shows that most of the students would agree or strongly agree with the statement. Other statements like "if they easily remembered how to use it" and "if they quickly became skillful with it" have a mean value of 6.76 and 6.62 respectively which shows that most of the students strongly agree with easiness of handling the BaghLearn application.

Last section (Table 4) was about participants' general opinion on the BaghLearn application. Participants were asked if they would recommend the BaghLearn application to other students in which the result obtained has a strong mean value of 6.52 and a standard deviation of 0.671 thus suggesting that they would strongly recommend it to their peers. Likewise, with a mean value of 6.38 and a corresponding standard deviation of 0.679 , most of the participants strongly felt the need to have it. while a mean value of 6.12 and 6.79 with a standard deviation of 0.772 and 0.425 respectively further suggests that most of the participants agreed with the fact that they had a wonderful experience and fun learning with the application.

\subsection{Qualitative results}

Individualized interview was organized as part of qualitative analysis that further helped to analyze students' development regarding the usability and effectiveness of

Table 3 Results showing the ease of learning with BaghLearn mobile game-based learning application

\begin{tabular}{lll}
\hline Statements & Mean & Standard Deviation \\
\hline Q20. one learned to use it quickly & 6.19 & 0.833 \\
Q21. one easily remembered how to use it & 6.76 & 0.431 \\
Q22. It is easy to learn to use it & 6.67 & 0.612 \\
Q23. One can quickly became skillful with it & 6.62 & 0.623 \\
\hline
\end{tabular}


Table 4 Results indicating the satisfiability of BaghLearn mobile game-based learning application

\begin{tabular}{lll}
\hline Statements & Mean & Standard Deviation \\
\hline Q24. I am satisfied with it & 6.14 & 1.072 \\
Q25. one would recommend it to a friend & 6.52 & 0.671 \\
Q26. It is fun to use & 6.79 & 0.415 \\
Q27. It works the way one wants it to work & 6.19 & 0.994 \\
Q28. It is wonderful & 6.12 & 0.772 \\
Q29. one felt the need to have it & 6.38 & 0.679 \\
\hline
\end{tabular}

the BaghLearn application. Since participating students were on a divergent level of knowledge and skills, this analysis would be beneficial in stretch of participants' diverse understanding on background and experiences they had while taking part in this research. Majority of the participants well-supported and found that the BaghLearn application will help them expand their problem solving ability which can be seen from quantitative analysis. This analysis further connects the quantitative result across the four themes yielded from the participant's assessment towards the BaghLearn application.

Fun game play All of the participant's interview thought BaghLearn has potential to boost motivation compared to what existing game patterns have. This application supports high scores and bonus quest that adds incentive to learners in order to keep them motivated which helps them to win the game easily. "In my point of view, BaghLearn is really good to bring up the fun of learning to students who want to learn Design and Analysis Algorithms, I scored higher in learning which helped me win comfortably over my opponent". "BaghLearn has that edge over other game-based learning applications as this does not feature a customized environment where game objects behavior is coded with the help of a code editor and a screen which replicates the effect of the code". These lines from participants reflects the fun learning structure of the application.

Better learning experiences People generally fails to recall just by hearing or reading, and are advised for some kind of practical training. Having said so, BaghLearn offers a shift in this regard, providing a new digital approach of learning from traditional face to face method. "I am a gamer; this application meets my need of playing along with learning. Apart from that I can learn from anywhere and anytime". "I can learn even if I miss classes for any reason since it features wider topics of algorithms". "The emphasis of the program on engaged learning made it even more appealing to use". "structure of the course content and the delivery method provided a nice fit" are few of the statements given by participants that supports better learning experience provided by BaghLearn application.

Interactive learning environment Participants felt engaged with learning material. They felt certain aspects of the game to be more interactive considering students can advance around at their will within the course component and even letting them with 
the option to skip if they wish to. Such features give students' some freedom to enjoy through the course and at their own pace. These statements given by the participants justify this theme: "I have a choice to learn what I want to. It has a learning button separately in case I prefer only to learn". "For me, this application is interesting to play. The game instructions are easy to perceive and I think the user interface is smooth and not excessively done".

Support and assistance Although it is not a common practice, few of the students urged the use of mobile devices freely in the classroom. "Universities should accept mobile learning as a support system in higher education learning". Few participants even acknowledged - "this application assist in preparation for their exam". While some confessed that BaghLearn is good for memory retention since it is a repetitive learning. Some even believe in providing support training, integration of ICT and practices of mobile learning on a broader scale so that, face to face learning is enriched with the use of mobile learning.

\section{Discussion}

This study was aimed at assessing BaghLearn mobile game-based application, implemented within a resource-constrained environment in the context of Nepalese Higher education system. This is due to the fact that resource-constrained environment games are often said to be less sophisticated compared to those in higher technical settings. So, the objective was to come up with a very simple game with minimal resource use and that includes learners' attributes.

In order to answer the research question, "To what extent do the students find the BaghLearn mobile game-based application to support computing education usable?" BaghLearn was evaluated and investigated using a sequential mixed method where both quantitative and qualitative data were analyzed to explain the perception of students towards BaghLearn providing support in computing education. The results indicated that BaghLearn not only improves learning performance in computing education but also support on several other important attributes of learning. The understanding and interpretation of students employing the use of BaghLearn for computing education has given significantly better learning experiences than those who were not accustomed to BaghLearn to assist their learning. After all, familiar nature of the application with Nepalese context cannot be neglected to ensure a better learning experience. Therefore, through the perceived learners' impression and judgement, it is clear that the BaghLearn application has made a positive advancement on learning dynamics. In other words, learners' exposure to the gamified learning platform has perceived positively regarding the delivery of design and analysis of algorithmic education relative to those who are from traditional teaching type (Haruna et al. 2019).

The main focus of BaghLearn is primarily to inspire learners crave for structured learning on subject matter where students feel comfortable to be involved in the learning process even if they are not in the classroom. The analysis on learning performance scores indicated that the BaghLearn application has supported this research and outperformed student's expectations. BaghLearn as a learning tool is found 
to improve students' ability in the course of design and analysis of algorithm with increased level of coherent knowledge. Students can learn anytime and anywhere either they are travelling, at home, at college or any other places and this learning setup is found to further helps students to systemize and implement the normal learning behavior even when they are not in their usual learning setup or a learning environment (Oyelere et al. 2018. Considering these findings, it shows that BaghLearn has improved competency among students of Nepalese Higher Education towards the study of design and analysis of algorithms.

Besides, the independent qualitative analysis among students regarding the impression and attitude towards mobile learning further supports the fact that there is a better positive perception of algorithmic course because of BaghLearn. From this observation we can say that, self-study became more visible and deeper (Oyelere et al. 2018; Shyshkanova et al. 2017), thus helping to recognize and annihilate the gap. Hence, deducing from our study we can say that, BaghLearn was able to improve the learning experience of students, bringing about positive impression and perception. Furthermore, due to a small study period and seemingly small number of participants, this study may not have a well-established observation and would require a comprehensive experiment for thorough implementation and instruction for higher education system.

\section{Conclusion}

BaghLearn mobile game-based application was developed to motivate learners from giving a boost to their algorithmic learning outcome in context to Nepalese Higher Education setting and with a very simple tech setting. The inspiration behind the development has been the rising acceptance of digital learning platforms, its highly interactive among the peer learners, affordability and most importantly the knowledge gain method which is better than the traditional teaching. The developed game is kept easy, without any complexity in order to serve in limited settings such that learner with a limited digital knowledge can entertain. Identifying the requirement, the BaghLearn application was designed and developed with the intention to provide an interactive learning environment with blended learning functionality between gaming and learning. Application has two modules - learning module and the game module, which is blended in such a way that it renders reminiscence. Learning functionality has a wide variety of topics followed by a series of notes in order to gain a positive impression towards learning. Students can even check their knowledge on the topic with enumerative quizzes. This application has a potential to improve algorithm learning experience since this platform benefits from the combination of motivation and engagement which deduce to enhanced knowledge gain.

The game-based learning platform "BaghLearn" has been designed and come up in cycle of development, test and improvement, the settings in which this platform is assessed best fits for it. Additionally, our theory towards BaghLearn was further supported by experimental evidence that was carried out to evaluate thus validating positive understanding among students towards game-based learning in Nepalese higher education system. 
On exploring the wide response, we accrue from the experience towards the use of BaghLearn, there is certainly an urge among students to ascertain the demand for digital learning and its incorporation within the university to support this kind of featured learning. Making use of technology in teaching is not an end as such. "How and what students learn with their mobile devices largely depends on how technology is integrated to support teaching and learning processes with the technology" (Parajuli 2016). There should be proper planning from the department itself regarding the teaching practice that urges to make use of mobile learning besides face to face learning thus embracing its conclusive association with learning performance. Hence, from the research it can be achieved that game-based learning application "BaghLearn" had helped learners to progress in their learning along with improvement in their cognitive behavior against algorithmic discipline.

\section{Limitations \& future work}

Despite having positive feedback from the students, there are certain limitations in this study, which is proposed to address in the future work. First, the small participant size could undermine the generalizability of the findings. Additionally, this research was based on Nepalese higher education system where gamebased learning is still a novice proposition, thus, the BaghLearn system may not be concluded in a global outreach. For our future work, the effort should be more adaptive to the course structure, in such a way that abstraction from a game concept to a realistic example can be a legitimate jump. Accompanied by this, we can add more practicability for learners in gaining better understanding of precise concepts. It is widely acknowledged that students in Nepalese society are more habitual to traditional methods of learning (i.e. face to face in a classroom), hence there is a need for understanding towards mobile learning. Having said so, the real changes can be seen only by practice. There should be a proper environment towards mobile learning practices within the institution. More robust framework is needed for mobile learning practices within and outside of the classroom along with extensive assessment and learning methods. A culture of sharing of information and resources should be developed. As part of our future research, this study should further extend to wider features of the BaghLearn application where teachers could send assignment, give feedback, share class lectures with students with focus on processing of information rather than limiting itself to possession of information. Additionally, we propose to test the application in contrast to the traditional teaching method to evaluate the learning outcome of the program, for example testing the application with two separate group of students and reviewing the learning result. Other future prospect of BaghLearn could be exploring the possibility of Virtual Reality in learning Algorithm that would provide more immersive feature with outstanding visualization that is not possible in the traditional classroom.

Acknowledgments Special thanks to the staff and students of Tribhuvan University, Institute of Engineering, Kathmandu, Nepal for participating in this study. The work by the second author, Solomon Sunday Oyelere was supported by the Jenny and Antti Wihuri Foundation, Finland. 
Funding Open access funding provided by University of Eastern Finland (UEF) including Kuopio University Hospital.

Open Access This article is licensed under a Creative Commons Attribution 4.0 International License, which permits use, sharing, adaptation, distribution and reproduction in any medium or format, as long as you give appropriate credit to the original author(s) and the source, provide a link to the Creative Commons licence, and indicate if changes were made. The images or other third party material in this article are included in the article's Creative Commons licence, unless indicated otherwise in a credit line to the material. If material is not included in the article's Creative Commons licence and your intended use is not permitted by statutory regulation or exceeds the permitted use, you will need to obtain permission directly from the copyright holder. To view a copy of this licence, visit http://creativecommons.org/licenses/by/4.0/.

\section{References}

Alkhalifah, T., Vries, D. \& Rampersad, G. (2017). Mobile learning adoption in developing countries. In proceedings of the 9th International Conference on Education Technology and Computers (ICETC 2017) (pp. 89-93). New York, NY: Association for Computing Machinery. https://doi.org/10.1145/ 3175536.3175583 .

All, A., Castellar, E., Castellar, N., \& Looy, J. (2016). Assessing the effectiveness of digital game-based learning: Best practices. Computers \& Education, 92, 90-103.

Anohah, E., Oyelere, S. S., Suhonen, J., \& Sutinen, E. (2017). Trends of mobile learning in computing education from 2006 to 2014: A systematic review of research publications. International Journal of Mobile and Blended Learning, 9(1), 16-33.

Antonellis, I., Bouras, C., Kapoulas, V., \& Poulopoulos, V. (2005). Design and Implementation of a Gamebased Learning Related Community. In Proceedings of Web Based Communities 2005 (IADIS).

Bada, \& Olusegun, S. (2015). Constructivism learning theory: A paradigm for teaching and learning. IOSR Journal of Research \& Method in Education, 5(6), 66-70.

Bartel, A., \& Hagel, G. (2014). Engaging students with a mobile game-based learning system in university education. International Journal of Interactive Mobile Technologies, 8(4), 56-60.

Carvalho, M. B., Bellotti, F., Berta, R., De Gloria, A., Sedano, C. I., Hauge, J. B., Hu, J., et al. (2015). An activity theory-based model for serious games analysis and conceptual design. Computers and Education, $87,166-181$.

Creswell, J, W., \& Clark, V, P, L. (2017). Designing and conducting mixed methods research, (3rd ed.), (pp. 520). SAGE Publications, Inc.

Creswell, J, W., Shope, R., Clark, V, L, P., \& Green, D, O. (2006). How interpretive qualitative research extends mixed methods research. Research in the schools, 13(1), 1-11.

Daniels, M., \& Pears, A. (2012). Models and methods for computing education research. In Proceedings of the 14th Australasian computing education conference (ACE2012) (pp. 95-102). Melbourne: ACM.

Daungcharone, K. (2016). Using Mobile game to enhance the learning motivation and performance in higher education. In K. Kim, N. Wattanapongsakorn, \& N. Joukov (Eds.), Mobile and wireless technologies 2016: Lecture notes in electrical engineering (pp. 215-222). Singapore: Springer.

Doran, K., Chaffin, A., Hicks, D., \& Barnes, T. (2009). Experimental evaluation of teaching recursion in a video game. In Proceedings of the 2009 ACM SIGGRAPH symposium on video games (sandbox '09) (pp. 79-86). New York: ACM.

Emerson, A., Sawyer, R., Azevedo, R. \& Lester, J. (2018). Gaze-enhanced student modeling for game-based learning. In proceedings of the 26th International Conference on Modeling, Adaptive and Personalization (UMAP'18) (pp. 63-72). New York, NY: Association for Computing Machinery. https://doi.org/10.1145/ 3209219.3209238.

Goldie, J. G. S. (2016). Connectivism: A knowledge learning theory for the digital age? Medical Teacher, 38(10), 1064-1069.

Guo, H. (2018). Application of a computer-assisted instruction system based on constructivism. International Journal of Emerging Technologies in Learning (iJET), 13(4), 33-44.

Hamari, J., Shernoff, D. J., Rowe, E., Coller, B., Asbell-Clarke, J., \& Edwards, T. (2016). Challenging games help students learn: An empirical study on engagement, flow and immersion in game-based learning. Computers in Human Behavior, 54, 170-179. 
Hartt, M., \& Mostafapour, M. (2019). Learning IS Child's play: Game-based learning in computer science education. ACM Transactions on Computing Education., 19(3), 1-18.

Haruna, H., Zainuddin, Z., Mellecker, R. R., Chu, S. K. W., \& Hu, X. (2019). An iterative process for developing digital gamified-sexual health education for adolescent students in low-tech settings. Information and Learning Sciences, 120(11/12), 723-742.

Hayati, A., Jalilifar, A., \& Mashhadi, A. (2013). Using short message service (SMS) to teach English idioms to EFL students. British Journal of Educational Technology, 44(1), 66-81.

Huang, Y., Chang, D., \& Wu, B. (2017). Mobile game-based learning with a Mobile app: Motivational effects and learning performance. Journal of Advanced Computational Intelligence and Intelligent Informatics, $21,963-970$.

Kachur, R., Mesnick, J., Liddon, N., Kapsimalis, C., Habel, M., \& David-Ferdon, C. et al. (2013). Adolescents, technology and reducing risk for HIV, STDs and Pregnancy. Centers for Disease Control and Prevention. Atlanta, GA: Centers for Disease Control and Prevention.

Kadel, R., Halder, S, J., Paudel, K, P., \& Gurung, M, P. (2018). Analyzing effect of GBL on student engagement and academic performance in computer networking course. In proceedings of the 20th International Conference on Information Integration and Web-based Applications \& Services (iiWAS2018) (pp. 143-145) New York, NY: Association for Computing Machinery. https://doi.org/10. $1145 / 3282373.3282855$.

Keskin, N. O., \& Metcalf, D. (2011). The current perspectives, theories and practices of Mobile learning. Turkish Online Journal of Educational Technology, 10(2), 202-208.

Khenissi, M. A., Essalmi, F., Jemni, M., \& Kinshuk, D. (2015). Learner modeling using educational games: A review of the literature. Smart Learning Environments, 2(1), 1-16.

Kinshuk, D., \& Chen, J. (2005). Mobile Technology in Educational Services. Journal of Educational Multimedia and Hypermedia, 14(1), 89-107.

Knobelsdorf, M. (2015). The theory behind theory - computer science education research through the lenses of situated learning. In A. Brodnik \& J. Vahrenhold (Eds.), Informatics in schools. Curricula, competences, and competitions. ISSEP 2015. Lecture notes in computer science (pp. 12-21). Cham: Springer.

Kukulska-Hulme, A., \& Shield, L. (2008). An overview of mobile assisted language learning: From content delivery to supported collaboration and interaction. ReCALL, 20(3), 271-289.

Lee, J., \& Hammer, J. (2011). Gamification in education: What, how, why bother? Academic Exchange Quarterly., 15(2), 1-5.

Lepp, A., Barkley, J., \& Karpinski, A. (2015). The relationship between cell phone use and academic performance in a sample of U.S. college students. SAGE Open, 5(1), 1-9.

Lund, A. M. (2001). Measuring usability with the USE questionnaire. Usability Interface, 8(2), 3-6.

Malliarakis, C., Satratzemi, M., Xinogalos, S. (2013). A holistic framework for the development of an educational game aiming to teach computer programming. In Proceeding of the European Conference on Games Based Learning in Porto, Portugal, (1), 359-368.

Malliarakis, C., Satratzemi, M., \& Xinogalos, S. (2014). Designing educational games for computer programming: A holistic framework. The Electronic Journal of e-Learning, 12(3), 281-181.

Mehdipour, Y., \& Zerehkafi, H. (2013). Mobile learning for education: Benefits and challenges. International Journal of Computational Engineering Research, 3(6), 93-100.

Ministry of Education. (2013). ICT in education: Master plan (2013-2017). Kathmandu: Ministry of Education https://moe.gov.np/assets/uploads/files/ICT_MP_2013_(Final).pdf. Accessed 26 January 2020.

Nepal Telecommunications Authority. (2020). Management information system. https://nta.gov.np/wpcontent/uploads/MIS-2076-KARTIK.p/. Accessed 28 January 2020.

ODEC-TU. (2015). Open and Distance Education Centre in Higher Education. ODECTU. http://www. odectu.edu.np/FrontendController/introduction/. Accessed 30 January 2020.

Oyelere S, S., Suhonen J, Laine T, H. (2017). Integrating Parson's programming puzzles into a game-based mobile learning application. In proceedings of the 17th Koli Calling International Conference on Computing Education Research, (pp. 158-162). New York, NY: Association for Computing Machinery. https://doi.org/10.1145/3141880.3141882.

Oyelere, S. S., Suhonen, J., Wajiga, G. M., \& Sutinen, E. (2018). Design, development, and evaluation of a mobile learning application for computing education. Education and Information Technologies, 23(1), $67-495$.

Parajuli, K. P. (2016). Mobile learning practice in higher education in Nepal. Open Praxis, 8(1), 41-54.

Parsons, G. (2010). Information provision for HE distance learners using mobile devices. The Electronic Library, 28(2), 231-244.

Prensky, M. (2002). The motivation of gameplay: The real twenty-first century learning revolution. On the Horizon, 10(1), 5-11. 
Resnick, M., Maloney, J., Monroy-Hernández, A., Rusk, N., Eastmond, E., Brennan, K., Millner, A., Rosenbaum, E., Silver, J., Silverman, B., \& Kafai, Y. (2009). Scratch: Programming for all. Communications of the ACM, 52(11), 60-67.

Rice, R. (2018). Implementing Connectivist teaching strategies in traditional K-12 classrooms. In F. H. Nah \& B. Xiao (Eds.), HCI in business, government, and organizations (pp. 645-655). Cham: Springer.

Richter, H., Barnes, T., Powell, E., Chaffin, A., \& Godwin, A. (2007). Game2Learn: Building CS1 learning games for retention. In Proceedings of the 12th Annual SIGCSE conference on Innovation and technology in computer science education (ITiCSE '07). (pp. 121-125). New York, NY: Association for Computing Machinery. https://doi.org/10.1145/1268784.1268821.

Rondon, S., Sassi, F, C., \& Andrade, C, R. (2013). Computer game-based and traditional learning method: A comparison regarding students' knowledge retention. BMC medical education,13(30). https://doi.org/10. 1186/1472-6920-13-30.

Sari, E., Tedjasaputra, A. (2019). Mobile learning: Enhancing social learning amongst millennials. In proceedings of Proceedings of Asian CHI Symposium 2019: Emerging HCI research collection (AsianHCI'19) (pp. 153-160). New York, NY: Association for Computing Machinery. https://doi.org/ 10.1145/3309700.3338460.

Schmitz, B., Klemke, R., Walhout, J., \& Specht, M. (2015). Attuning a mobile simulation game for school children using a design-based research approach. Computer \& Education, 81, 35-48.

Sharples, M., Taylor, J., \& Vavoula, G. (2007). A theory of learning for the mobile age. In R. Andrews \& C. Haythornthwaite (Eds.), The Sage handbook of E-learning research (pp. 221-247). London: SAGE Publications Ltd..

Shonola, S. A., Joy, M. S., Oyelere, S. S., \& Suhonen, J. (2016). The impact of mobile devices for learning in higher education institutions: Nigerian universities case study. International Journal of Modern Education and Computer Science, 8(8), 43-50.

Shyshkanova, G., Zaytseva, T., \& Frydman, O. (2017). Mobile technologies make education a part of everyday life. Information and Learning Sciences, 118(11/12), 570-582.

Taylor, E., Breed, M., Hauman, I. \& Homann, A. (2013). Choosing learning methods suitable for teaching and learning in computer science. International Association for Development of the Information Society, 7482.

Tewari, A., Kumar, A., Shroff, G., Chittamuru, D., Kam, M., \& Canny, J. (2010). An exploratory study of unsupervised mobile learning in rural India. In Proceedings of the SIGCHI conference on human factors in computing systems (CHI '10) (pp. 743-752). New York: ACM.

Tlili, A., Essalmi, F., \& Jemni, M. (2016a). Metric-based approach for selecting the game genre to model personality. In Y. Li et al. (Eds.), State-of-the-art and future directions of smart learning: Lecture notes in educational technology (pp. 275-279). Singapore: Springer.

Tlili, A., Essalmi, F., \& Jemni, M. (2016b). Improving learning computer architecture through an educational mobile game. Smart Learning Environments, 3(7), 1-14.

Tsai, C., Cheng, C., Yeh, D., \& Lin, S. (2017). Can learning motivation predict learning achievement? A case study of a mobile game-based English learning approach. Education and Information Technologies, 22(5), 2159-2173.

Vasudeva, S., Colthorpe, K. \& Ernst, H. (2017). Student-initiated Mobile Learning in Higher Education. In proceedings of the 16th World Conference on Mobile and Contextual Learning (mLearn 2017) (pp. 14). New York, NY: Association for Computing Machinery.

Zendler, A. (2018). Instructional methods in computing education judged by computer science teachers and educational experts. it-Information. Technology, 60(2), 79-90.

Zhu, J., Alderfer, K., Furqan, A., Nebolsky, J., Char, B., Smith, B., Villareale, J. \& Ontanon, S. (2019). Programming in game space: How to represent parallel programming concepts in an educational game. In proceedings of the 14th International Conference on the Foundation of Digital Games (FDG '19). New York, NY: Association for Computing Machinery. (4), 1-10. https://doi.org/10.1145/3337722.3337749.

Publisher's note Springer Nature remains neutral with regard to jurisdictional claims in published maps and institutional affiliations. 
Alok Kumar Yadav is a MSc. student in Computer Science from the University of Eastern Finland, Joensuu, Finland. He received his bachelor Degree (B. Tech) in Information Technology from Motilal Nehru National Institute of Technology, Allahabad, India. He is interested in educational technology, Human Language technology and computational thinking.

Solomon Sunday Oyelere received a $\mathrm{PhD}$ degree in Computer science from the University of Eastern Finland, Joensuu, Finland, MSc. degree in Research in Computer and Systems Engineering from Ilmenau University of Technology, Germany, and B. Tech (Honors) degree in Computer science from Federal University of Technology Yola-Nigeria. Solomon's research interest focuses mainly on improving learning environments through smart technology, pedagogy, and content. He applies techniques from interdisciplinary areas including computing education, educational technology, interactive mobile computing, educational sciences, web technology, and educational data mining. 